\title{
LIMIT CYCLES FOR CUBIC SYSTEMS WITH A SYMMETRY OF ORDER 4 AND WITHOUT INFINITE CRITICAL POINTS
}

\author{
M. J. ÁlVAREZ, A. GASUlL, AND R. PROHENS \\ (Communicated by Carmen C. Chicone)
}

\begin{abstract}
In this paper we study those cubic systems which are invariant under a rotation of $2 \pi / 4$ radians. They are written as $\dot{z}=\varepsilon z+p z^{2} \bar{z}-\bar{z}^{3}$, where $z$ is complex, the time is real, and $\varepsilon=\varepsilon_{1}+i \varepsilon_{2}, p=p_{1}+i p_{2}$ are complex parameters. When they have some critical points at infinity, i.e. $\left|p_{2}\right| \leq 1$, it is well-known that they can have at most one (hyperbolic) limit cycle which surrounds the origin. On the other hand when they have no critical points at infinity, i.e. $\left|p_{2}\right|>1$, there are examples exhibiting at least two limit cycles surrounding nine critical points. In this paper we give two criteria for proving in some cases uniqueness and hyperbolicity of the limit cycle that surrounds the origin. Our results apply to systems having a limit cycle that surrounds either 1,5 or 9 critical points, the origin being one of these points. The key point of our approach is the use of Abel equations.
\end{abstract}

\section{INTRODUCTION AND MAIN RESULTS}

This paper deals with the equation

$$
\dot{z}=\frac{d z}{d t}=\varepsilon z+p z^{2} \bar{z}-\bar{z}^{3},
$$

where $z$ is a point in the complex plane, $t$ is real, $\varepsilon=\varepsilon_{1}+i \varepsilon_{2}, p=p_{1}+i p_{2}$ are complex parameters, and $p_{2} \geq 0$. In Section 2 we will see that this last inequality can be assumed without loss of generality. We are interested in the number and location of the limit cycles of this equation that surround the origin and, eventually, other critical points.

The previous equation (1.1) is the particular case for $q=4$ of a general family, the one with a rotational invariance of $2 \pi / q$ radians $(q \in \mathbb{N}, q>2)$. In [2] it is proved that the differential equations which are invariant for a rotation of an angle of $2 \pi / q$ radians have the normal form

$$
\dot{z}=z \mathcal{A}\left(|z|^{2}\right)+\mathcal{B} \bar{z}^{q-1}+O\left(|z|^{q+1}\right), q>2,
$$

Received by the editors March 24, 2006 and, in revised form, January 16, 2007.

2000 Mathematics Subject Classification. Primary 34C07, 34C14; Secondary 34C23, 37C27.

Key words and phrases. Planar autonomous ordinary differential equations, symmetric cubic systems, limit cycles.

The first two authors were partially supported by grants MTM2005-06098-C02-1 and 2005SGR00550. The third author was supported by grant UIB-2006. This paper was also supported by the CRM Research Program: On Hilbert's 16th Problem. 
where $\mathcal{A}$ is a complex polynomial of degree $[(q-1) / 2]$ and $\mathcal{B}$ is a non-zero complex constant. The above equation without the remainder term is called the principal equation. For instance, the principal equations for the particular cases $q=3,4$ are, respectively,

$$
\dot{z}=a_{0} z+a_{1} z|z|^{2}+\mathcal{B} \bar{z}^{2}, \quad \dot{z}=a_{0} z+a_{1} z|z|^{2}+\mathcal{B} \bar{z}^{3} .
$$

The cases $q \leq 4$ are called strong resonances while the cases $q \geq 5$ are called weak resonances. The flow for all the cases of the family, except $q=4$, is studied in several places (see [10] for $q<4$ and [2] for $q>4$ ). The study of the periodic orbits of case $q=4$ turns out to be especially difficult. In the following we summarize several well-known partial results given in [2], [4], [6] (here can be found a complete study in the whole family (1.2)), [13] and [15]. The study of equation (1.1) can be split into the following three cases:

- Case 1: Equation (1.1) has a unique critical point, the origin.

- Case 2: Equation (1.1) has five critical points, the origin and four saddlenodes.

- Case 3: Equation (1.1) has nine critical points, the origin, four saddle points and four critical points of index +1 .

Case 1 is totally understood. In [4] it is proved that in this case, there are only two possibilities for the global phase portrait of the equation: either there exists a unique periodic orbit, and when it exists it is hyperbolic, or the origin is a global attractor. In that article the authors also completely solve the case where the Poincaré compactification of equation (1.1) has critical points at infinity. As we will see in Lemma 2.2, it corresponds to the condition $p_{2} \leq 1$. In this case, there exists at most one limit cycle encircling the origin and, when it exists, it is hyperbolic. Moreover, if there exist non-zero critical points they cannot be surrounded by a periodic orbit.

The problem of the number of limit cycles not surrounding the origin corresponds to Case 3 and it is also totally solved. In [15] it is proved that either there are no limit cycles or there are exactly four hyperbolic ones, each one of them surrounding exactly one of the critical points of index +1 .

Thus, the remaining problem is to determine, in the case where infinity has no critical points, the number of limit cycles that can exist surrounding the origin, and eventually surrounding also the other 4 or 8 critical points, and whether they coexist or not with the 4 limit cycles that do not surround the origin. In [6] and [13] it is proved that, in the remaining case $p_{2}>1$, at least two limit cycles can exist surrounding the 9 critical points. Both works consider the Hamiltonian system,

$$
\dot{z}=i \varepsilon_{2} z+i p_{2} z^{2} \bar{z}-\bar{z}^{3},
$$

having 9 critical points ( 5 centers and 4 saddles) in its phase portrait and a ring full of periodic orbits surrounding the 9 critical points. When this equation is perturbed to get a complete equation (1.1), it is proved that, at least, two of the periodic orbits of that ring give rise to two hyperbolic limit cycles for the perturbed equation. As far as we know, the last results appeared for this case are mainly numeric or have been proved assisted by a computer, see [14] and [9] respectively.

A full description of computer-generated pictures of all the phase portraits of equation (1.1) is given in [11].

Our main result is: 
Theorem 1.1. (a) Consider equation (1.1) with $\varepsilon_{2} \neq 0, p_{2}>1$ and define the following four numbers:

$$
\begin{aligned}
\Sigma_{A}^{-}=\frac{\varepsilon_{2} p_{1} p_{2}-\sqrt{\varepsilon_{2}^{2}\left(p_{1}^{2}+p_{2}^{2}-1\right)}}{p_{2}^{2}-1}, & \Sigma_{A}^{+}=\frac{\varepsilon_{2} p_{1} p_{2}+\sqrt{\varepsilon_{2}^{2}\left(p_{1}^{2}+p_{2}^{2}-1\right)}}{p_{2}^{2}-1}, \\
\Sigma_{B}^{-}=\frac{\varepsilon_{2} p_{1} p_{2}-\sqrt{\varepsilon_{2}^{2}\left(p_{1}^{2}+9 p_{2}^{2}-9\right)}}{2\left(p_{2}^{2}-1\right)}, & \Sigma_{B}^{+}=\frac{\varepsilon_{2} p_{1} p_{2}+\sqrt{\varepsilon_{2}^{2}\left(p_{1}^{2}+9 p_{2}^{2}-9\right)}}{2\left(p_{2}^{2}-1\right)} .
\end{aligned}
$$

If one of the conditions

$$
\text { (i) } \varepsilon_{1} \notin\left(\Sigma_{A}^{-}, \Sigma_{A}^{+}\right), \quad(\text { ii }) \quad \varepsilon_{1} \notin\left(\Sigma_{B}^{-}, \Sigma_{B}^{+}\right),
$$

is satisfied, then equation (1.1) has at most one limit cycle surrounding the origin. Furthermore, when it exists it is hyperbolic.

(b) There are equations (1.1) under condition (i) having exactly one hyperbolic limit cycle surrounding either 1 or 5 critical points and equations under condition (ii) having exactly one limit cycle surrounding either 1, 5 or 9 critical points.

Remark 1.2. In the case that $\varepsilon_{2}=0$ equation (1.1) can be integrated and it is not difficult to see that it has no periodic orbits.

A key point for our study is that equation (1.1) can be transformed into an Abel equation. Some of the known results about the number of limit cycles surrounding the origin of equation (1.1) can also be reproduced by using our approach which, as far as we know, has not been used before to study equation (1.1).

As we will see in Proposition 2.5, the results of Theorem 1.1 when condition (i) is satisfied apply only in Cases 1 and 2 defined above. Condition (ii) applies to a more interesting and difficult situation, namely Case 3, where the limit cycles surrounding the origin also could surround the other 8 critical points.

\section{Preliminary RESUlts}

A first reduction in the principal equation associated to equation (1.2) when $q=4$ is that $\mathcal{B}$ can be chosen to be real; in the literature it is found either $\mathcal{B}=1$ or $\mathcal{B}=-1$. We have set this last possibility in equation (1.1). Furthermore, as we have said before, we can always assume $p_{2} \geq 0$, by changing $z$ by $\bar{z}$, if necessary. Our first result reduces the study of the periodic orbits of equation (1.1) that surround the origin to the study of the number of solutions that satisfy $x(0)=x(2 \pi)$ of an Abel equation

$$
\frac{d x}{d \theta}=A(\theta) x^{3}+B(\theta) x^{2}+C(\theta) x,
$$

with $A, B$ and $C$ smooth, $2 \pi$-periodic functions. For these solutions their multiplicity can be defined as the usual multiplicity: as zeroes of the displacement map defined by the flow of equation (2.1) between $\theta=0$ and $\theta=2 \pi$. We prove the next result, which essentially follows from the results of [5].

Lemma 2.1. Each periodic orbit $\gamma$ of equation (1.1), with $\varepsilon_{2} \neq 0$, that surrounds the origin corresponds to a non-zero solution $\Gamma$ of the Abel equation (2.1) that satisfies $x(0)=x(2 \pi)$, where $C(\theta)=2 \varepsilon_{1} / \varepsilon_{2}$,

$$
\begin{aligned}
A(\theta) & =\frac{4}{\varepsilon_{2}}\left(p_{2}+\sin (4 \theta)\right)\left(\left(\cos (4 \theta)-p_{1}\right) \varepsilon_{2}+\left(p_{2}+\sin (4 \theta)\right) \varepsilon_{1}\right) \quad \text { and } \\
B(\theta) & =-\frac{2}{\varepsilon_{2}}\left(2 \varepsilon_{1} p_{2}-\varepsilon_{2} p_{1}+3 \varepsilon_{2} \cos (4 \theta)+2 \varepsilon_{1} \sin (4 \theta)\right) .
\end{aligned}
$$


Furthermore the multiplicity of the periodic orbit $\gamma$ coincides with the multiplicity of $\Gamma$.

Proof. We can transform equation (1.1) in the cylinder $\mathbb{R}^{+} \times[0,2 \pi]$ through the change of variables $z=\sqrt{r}(\cos (\theta)+i \sin (\theta))$, that is always well-defined because we are interested in $r \geq 0$. We obtain

$$
\left\{\begin{aligned}
\dot{r} & =2 \varepsilon_{1} r+2\left(p_{1}-\cos (4 \theta)\right) r^{2} \\
\dot{\theta} & =\varepsilon_{2}+\left(p_{2}+\sin (4 \theta)\right) r .
\end{aligned}\right.
$$

Note that the limit cycles surrounding the origin of (1.1) become periodic orbits of system (2.2) in the cylinder with $r>0$ and, as is proved in [7], they do not intersect the set $\left\{(r, \theta): \dot{\theta}=\varepsilon_{2}+\left(p_{2}+\sin (4 \theta)\right) r=0\right\}$. So they are solutions of

$$
\frac{d r}{d \theta}=\frac{2 \varepsilon_{1} r+2\left(p_{1}-\cos (4 \theta)\right) r^{2}}{\varepsilon_{2}+\left(p_{2}+\sin (4 \theta)\right) r},
$$

that satisfy $r(0)=r(2 \pi)$. As $\varepsilon_{2} \neq 0$, we can use Cherkas' transformation, $x=$ $r /\left(\varepsilon_{2}+\left(p_{2}+\sin (4 \theta)\right) r\right)$ (see [5]), and equation (2.3) is converted into the Abel equation (2.1). Moreover, all periodic orbits of system (1.1) are transformed into solutions of the Abel equation that satisfy $x(0)=x(2 \pi)$. It is also clear that Cherkas' transformation keeps the multiplicity of the solutions. Thus the result follows.

As we are mainly interested in the case that the infinity in the Poincaré compactification has no critical points (the other cases are already solved), we prove a lemma that provides us this condition in terms of the parameters of equation (1.1). Moreover, we get the stability of the infinity and the origin.

Lemma 2.2. Consider equation (1.1).

(i): Infinity in the Poincaré compactification has no critical points if and only if $p_{2}>1$.

(ii): When $p_{2}>1$, infinity is an attractor (resp. a repellor) when $p_{1}>0$ (resp. $\left.p_{1}<0\right)$.

(iii): The origin is an attractor (resp. a repellor) when $\varepsilon_{1}<0$ (resp. $\left.\varepsilon_{1}>0\right)$.

Proof. If in equation (2.2) we make the change of variable $R=1 / r$ and the rescaling of the time $\frac{d t}{d s}=R$, we get a polar version of the Poincaré compactification:

$$
\left\{\begin{array}{l}
R^{\prime}=-2\left(p_{1}-\cos (4 \theta)\right) R-2 \varepsilon_{1} R^{2} \\
\theta^{\prime}=p_{2}+\sin (4 \theta)+\varepsilon_{2} R
\end{array}\right.
$$

This system has no critical points on infinity, which is given by the invariant set $\{R=0\}$, if and only if $p_{2}>1$.

We want to compute the stability of infinity in the case $p_{2}>1$. Applying the results in [12], we have that the stability of $\{R=0\}$ in system (2.4) is given by the sign of

$$
\int_{0}^{2 \pi} \frac{-2\left(p_{1}-\cos (4 \theta)\right)}{p_{2}+\sin (4 \theta)} d \theta=\frac{-4 \pi p_{1}}{\sqrt{p_{2}^{2}-1}},
$$

and then the result follows. The study of the stability of the origin is trivial because it is a non-degenerate critical point.

We will need the following general result about Abel equations proved in [8], that we state for the sake of completeness. 
Theorem 2.3. Consider the Abel equation (2.1) and assume that either $A(\theta) \not \equiv 0$ or $B(\theta) \not \equiv 0$ does not change sign. Then it has at most three solutions satisfying $x(0)=x(2 \pi)$, taking into account their multiplicities.

The next lemma gives explicit conditions on $p$ and $\varepsilon$ to ensure that either the function $A$ or the function $B$, associated to equation (1.1), does not change sign.

Lemma 2.4. When $p_{2}>1$, consider the values $\Sigma_{A}^{+}, \Sigma_{A}^{-}, \Sigma_{B}^{+}$, and $\Sigma_{B}^{-}$defined in Theorem 1.1. Let $A(\theta)$ and $B(\theta)$ be the functions given in Lemma 2.1. Then

(i): The function $A(\theta)$ changes sign if and only if $\varepsilon_{1} \in\left(\Sigma_{A}^{-}, \Sigma_{A}^{+}\right)$,

(ii): The function $B(\theta)$ changes sign if and only if $\varepsilon_{1} \in\left(\Sigma_{B}^{-}, \Sigma_{B}^{+}\right)$.

Proof. (i) It is not difficult to check that $A(\theta)$ changes sign only for the values of $\varepsilon_{1}$ stated above. A sketch of the verification is as follows. We compute the maxima and the minima of the function $A(\theta)$. As $p_{2}>1$ it is equivalent to compute the extrema of $\left(\cos (4 \theta)-p_{1}\right) \varepsilon_{2}+\left(p_{2}+\sin (4 \theta)\right) \varepsilon_{1}$. We apply the change of variables $\cos (4 \theta)=x$ and $\sin (4 \theta)=y$, and we optimize the function subject to the restriction $x^{2}+y^{2}=1$. We get that the value of the function at both extrema is $A_{ \pm}=$ $\varepsilon_{1} p_{2}-\varepsilon_{2} p_{1} \pm \sqrt{\varepsilon_{1}^{2}+\varepsilon_{2}^{2}}$. They are both non-negative or non-positive if and only if $\varepsilon_{1} \in\left(-\infty, \Sigma_{A}^{-}\right] \cup\left[\Sigma_{A}^{+},+\infty\right)$. The proof of (ii) follows similarly.

The next result gives a geometric interpretation of the behavior of the function $A(\theta)$ appearing in the Abel equation (2.1) in terms of the number of critical points of equation (1.1).

Proposition 2.5. Consider equation (1.1) and assume that $\varepsilon_{2} \neq 0$ and $p_{2}>1$. Define $\Theta=\left\{(r, \theta): \dot{\theta}=\varepsilon_{2}+\left(p_{2}+\sin (4 \theta)\right) r=0\right\}$. Then

(i): If $\Theta=\emptyset$, then equation (1.1) has only one critical point, the origin.

(ii): When $\Theta \neq \emptyset$, the set $\Theta$ is a closed curve that surrounds the origin. Furthermore:

(a): If for all $\theta \in[0,2 \pi], \varepsilon_{2} A(\theta)>0$ (resp. $<0$ ), then equation (1.1) has only one critical point, the origin, and the interior of $\Theta$ is negatively (resp. positively) invariant.

(b): If for all $\theta \in[0,2 \pi], \varepsilon_{2} A(\theta) \geq 0$ (resp. $\leq 0$ ), then equation (1.1) has five critical points and the vector field associated to the differential equation (1.1) points towards the exterior (resp. interior) of the region delimited by $\Theta$ at all points of this curve except at the four critical points.

(c): If the function $A(\theta)$ changes sign, then equation (1.1) has nine critical points.

Proof. Following the proof of Lemma 2.1 we write equation (1.1) as (2.2). The proof of (i) is trivial.

(ii) The non-zero critical points are the points $(r, \theta)$, with $r>0$, that belong to $\{\dot{\theta}=0\} \cap\{\dot{r}=0\}$. Since $p_{2}+\sin (4 \theta) \neq 0$, the set $\Theta$ is given by the closed curve $r=-\varepsilon_{2} /\left(p_{2}+\sin (4 \theta)\right)$. If we compute $\dot{r}$ on $\Theta$ we obtain

$$
\left.\dot{r}\right|_{\dot{\theta}=0}=r \frac{\varepsilon_{2} A(\theta)}{\left(p_{2}+\sin (4 \theta)\right)^{2}} .
$$

From the above equality the lemma follows. 


\section{Proof of Theorem 1.1}

Let us define the function $c(\theta)=p_{2}+\sin (4 \theta)$. Observe that, as $p_{2}>1, c(\theta)>0$ for all $\theta \in[0,2 \pi]$.

(a) We start assuming that condition (i) is satisfied. By using Lemma 2.1 we know that the study of the periodic orbits of equation (1.1) can be reduced to the study of the periodic orbits of the Abel equation (2.1). Furthermore, since $\varepsilon_{1} \notin\left(\Sigma_{A}^{-}, \Sigma_{A}^{+}\right)$, by condition (i) of Lemma 2.4 we know that the function $A(\theta)$ appearing in the Abel equation does not change sign. Hence, from Theorem 2.3, the maximum number of solutions satisfying $x(0)=x(2 \pi)$ in system $(2.1)$, taking into account their multiplicity, is three. One of them is $x=0$. Since $c(\theta) \neq 0$, by some simple calculations it is easy to prove that the curve $x=1 / c(\theta)$ is a second solution satisfying this condition. If we undo the Cherkas' transformation used to convert equation (2.3) into the Abel equation, it turns out that $x=1 / c(\theta)$ is mapped into the infinity of the differential equation. Then, by Lemma 2.1, the maximum number of limit cycles of equation (1.1) is one. The fact that this limit cycle, when it exists, is hyperbolic, also follows from the same lemma.

The proof of (a) when condition (ii) is satisfied follows the same steps, but it changes the role of $A(\theta)$ by the role of $B(\theta)$.

(b) We fix the parameters of equation (1.1) as: $\varepsilon_{2}=-1, p_{1}=-1 / 2$ and $p_{2}=5 / 4$. In this case, $\Sigma_{A}^{+}=\frac{2}{9}(5+2 \sqrt{13})$, and $\Sigma_{B}^{+}=\frac{1}{9}(5+2 \sqrt{85})<\Sigma_{A}^{+}$.

We study the family for $\varepsilon_{1}>\Sigma_{A}^{+}$. Applying Lemma 2.2, the stability of the origin is given by the sign of $\varepsilon_{1}>0$, hence it is a strong unstable focus. By the same lemma, we get that the infinity in the Poincaré compactification is also unstable.

From Lemma 2.4, when $\varepsilon_{1}>\Sigma_{A}^{+}, A(\theta)$ does not vanish and there are no other critical points but the origin. Furthermore, since $\varepsilon_{2} A(\theta)>0$, by using Proposition 2.5(ii) (a) we know that the exterior of the closed curve $\Theta$ is positively invariant. Hence by using the Poincaré-Bendixson theorem, the behavior at infinity and part (a) of this theorem, we know that the differential equation has exactly one limit cycle which surrounds the curve $\Theta$ and that it is hyperbolic and stable.

When $\varepsilon_{1}=\Sigma_{A}^{+}$, four semi-elementary critical points appear on $\Theta$. By using $[1$, Thm.65], it is not difficult to check that all of them are saddle-nodes. We are going to prove that, at this value of the parameter $\varepsilon_{1}$, the periodic orbit still exists and surrounds the five critical points. Since infinity is always a repellor, the only way for this limit cycle to disappear is going, when $\varepsilon_{1}$ goes to $\Sigma_{A}^{+}$, to a limit periodic set formed by the four saddle-nodes and their separatrices. To prevent this situation we will construct a polygonal line without any contact point with the flow of the differential equation, which will help us to control the behavior of the separatrices of the four saddle-nodes. More concretely, this polygonal line forces the unstable separatrices of the saddle-nodes to have as its $\omega$-limit a periodic orbit surrounding $\Theta$; see Figure 1.

Because of the symmetry of the system it is enough to study one of the saddlenodes, for instance the one in the first quadrant, namely $\left(x_{0}, y_{0}\right)$, in cartesian coordinates. 


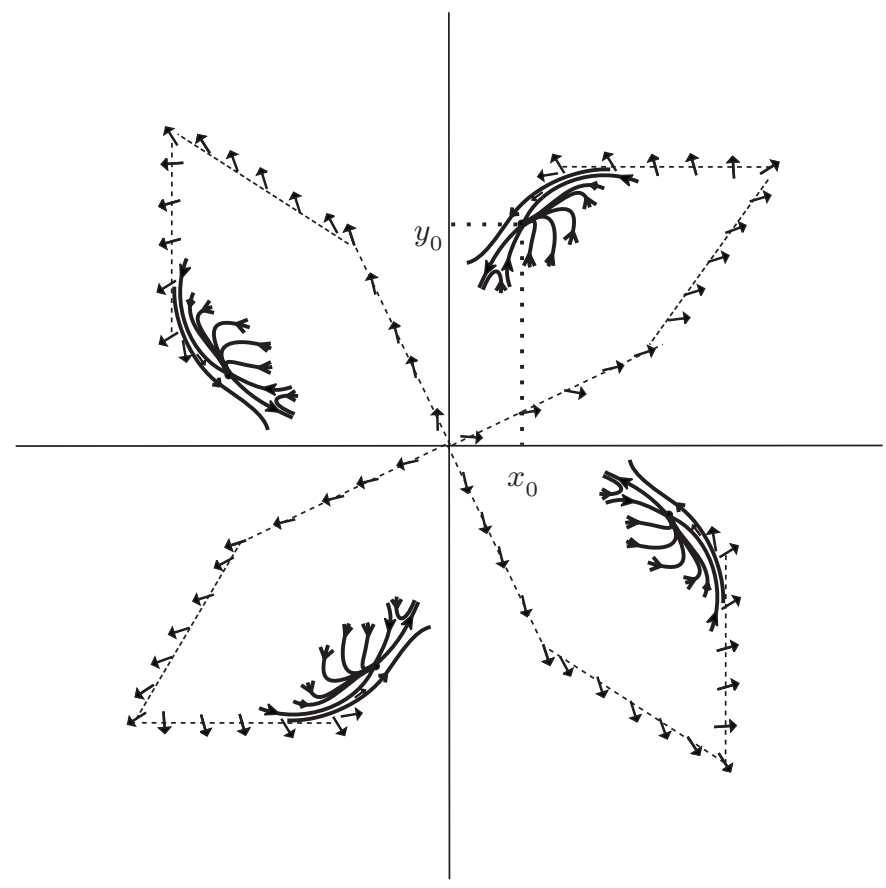

Figure 1. The polygonal curve without any contact and the separatrices of the saddle-node of system (2.2).

Let $x_{1}$ be the unique real zero of the equation

$$
\begin{array}{r}
\frac{5}{4} x^{3}+\frac{3}{2}(-2+\sqrt{10}) x^{2}+\left(\frac{53}{10}-9 \sqrt{\frac{2}{5}}\right) x \\
+\frac{1}{375}(7762-2423 \sqrt{10}+100 \sqrt{13}(-2+\sqrt{10}))=0,
\end{array}
$$

that is, approximately, $x_{1} \simeq 0.56$, and consider the polygonal line defined as

$$
(x(t), y(t))= \begin{cases}(t, t), & \text { if } 0 \leq t<\sqrt{\frac{2}{5}} \\ \left(t, 3\left(t-\sqrt{\frac{2}{5}}\right)+\sqrt{\frac{2}{5}}\right), & \text { if } \sqrt{\frac{2}{5}} \leq t<1, \\ \left((t-1)\left(x_{1}-1\right)+1,3-2 \sqrt{\frac{2}{5}}\right), & \text { if } 1 \leq t<2, \\ \left((t-3)\left(x_{0}-x_{1}\right)+x_{0},\right. & \\ \left.(t-3)\left(y_{0}-3+2 \sqrt{\frac{2}{5}}\right)+y_{0}\right), & \text { if } 2 \leq t<3 .\end{cases}
$$

The flow associated to equation (2.2) is transversal to the previous polygonal line, that is, the polygonal does not have any contact point. We give the details for $\sqrt{\frac{2}{5}} \leq t<1$. The other cases follow by using analogous arguments.

Let us consider the explicit expression of the second segment of the polygonal line as $y=3\left(x-\sqrt{\frac{2}{5}}\right)+\sqrt{\frac{2}{5}}$. The scalar product of the vector field associated to 
equation (2.2) with the normal vector to the former segment, $(-3,1)$, is given by

$$
\frac{-2 \sqrt{\frac{2}{5}}(77+20 \sqrt{13})}{45}+\frac{62 x}{5}-59 \sqrt{\frac{2}{5}} x^{2}+29 x^{3},
$$

when it is evaluated on the segment. This cubic equation has only one real root $x_{2}>1$, which is simple. Hence, for $x \in\left[\sqrt{\frac{2}{5}}, 1\right]$ the scalar product is negative and, consequently, the flow crosses the considered segment transversally.

By using the previous arguments and the fact that infinity is a repellor, by the Poincaré-Bendixson theorem the only possible $\omega$-limit for the unstable separatrix of the saddle-node is a periodic orbit which has to surround the four saddle-nodes; see again Figure 1.

Since for the value $\varepsilon_{1}=\Sigma_{A}^{+}$, the function $A(\theta)$ does not change its sign, from part (a) of this theorem, we get the hyperbolicity of the limit cycle.

Now we move $\varepsilon_{1}$ a little more towards zero but very close to $\Sigma_{A}^{+}$. In this case, $B(\theta)$ is strictly positive because $\Sigma_{A}^{+}>\Sigma_{B}^{+}$and we have 9 critical points: four saddles and four critical points of index +1 on $\Theta$ and a focus at the origin. Applying again part (a) but now condition (ii), we know that the maximum number of limit cycles surrounding the origin is one. As for $\varepsilon_{1}=\Sigma_{A}^{+}$, the limit cycle was hyperbolic, it still exists. Then we have a vector field with $B(\theta)$ not changing sign, 8 non-zero critical points and a limit cycle surrounding them and the origin.

3.1. Some remarks on the proof of Theorem 1.1. (i) We note that in the proof of the above theorem, when $\varepsilon_{1}=\Sigma_{A}^{+}$, the vector field associated to equation (1.1) points towards the exterior of the region delimited by $\Theta$ at all points of this curve except at the four saddle-nodes. Nevertheless, one of the stable separatrices of each one of the saddle-nodes lies outside of the region bounded by $\Theta$. Hence, the behavior of the vector field on this curve is not enough to conclude that the limit cycle still exists for this value of $\varepsilon_{1}$ and we have needed to introduce the polygonal line used in the proof.

(ii) The fact that when $c(\theta)$ does not vanish the curve $x=1 / c(\theta)$ is a periodic orbit of the Abel equation (2.1) is not exclusive for this particular equation. In fact, it is valid for all the Abel equations obtained from the Cherkas' transformation applied to planar polynomial vector fields with homogeneous non-linearities; see for instance [3].

(iii) The same result stated as condition (i) of our main theorem but only when the function $A(\theta)$ does not vanish has also been proved in [4] by using different methods. Dynamically there is a big difference, because in our result allowing $A(\theta)$ to vanish but not changing sign means that equation (2.1) has exactly 5 critical points. On the other hand the results of [4] apply only when the critical point is unique.

(iv) Observe that the values of the parameters considered in the example given in (b) of the previous result are not close to any Hamiltonian system. Moreover, the concrete set of values of our example are in the region $\mathrm{C}$ of [6] and in the region (iii) of [4], the most difficult region to study, as said by Arnold in [2].

\section{ACKNOWLEDGMENT}

We are grateful to A. Zegeling for proposing that we study this problem. 


\section{REFERENCES}

1. Andronov, A.A.; Leontovich, E.A.; Gordon, I.I.; Maier, A.L., "Qualitative theory of second-order dynamic systems", John Wiley and Sons, New York, 1973. MR0350126 (50:2619)

2. Arnold, V., "Chapitres supplémentaires de la théorie des équations différentielles ordinaires", Éditions Mir-Moscou, 1980. MR626685 (83a:34003)

3. Carbonell, M.; Llibre, J. Limit cycles of polynomial systems with homogeneous nonlinearities. J. Math. Anal. Appl., 142 (1989), 573-590. MR1014597 (91d:58205)

4. Cheng, C.Q.; Sun, Y.S. Metamorphoses of phase portraits of vector filed in the case of symmetry of order 4. J. of Diff. Equations, 95 (1992), 130-139. MR1142279 (93a:58144)

5. Cherkas, L.A. Number of limit cycles of an autonomous second-order system. Diff. Equations, 5 (1976), 666-668.

6. Chow, S.N.; Li, C.; Wang, D., "Normal Forms and Bifurcation of Planar Vector Fields", Cambridge University Press, 1994. MR1290117 (95i:58161)

7. Coll, B.; Gasull, A.; Prohens, R. Differential equations defined by the sum of two quasihomogeneous vector fields. Can. J. Math., 49 (1997), 212-231. MR1447489 (98j:34041)

8. Gasull, A.; Llibre, J. Limit cycles for a class of Abel Equation. SIAM J. Math. Anal., 21 (1990), 1235-1244. MR1062402 (91e:34036)

9. Guckenheimer, J. Phase portraits of planar vector fields: computer proofs. Exp. Mathematics, 4 (1995), 153-165. MR1377416 (97g:34041)

10. Horozov, E. Versal deformations of equivariant vector fields for the case of symmetries of order 2 and 3. Trudy Sem. Pet., 5 (1979), 163-192 (in Russian). MR549627 (80k:58079)

11. Krauskopf, B. Bifurcation sequences at 1:4 resonance: an inventory. Nonlinearity, 7 (1994), 1073-1091. MR1275542 (95d:58097)

12. Lloyd, N.G. A note on the number of limit cycles in certain two-dimensional systems. J. London Math. Soc., 20 (1979), 277-286. MR551455 (80k:34039)

13. Neishtadt, A.I. Bifurcation of the phase pattern of an equation system arising in the problem of stability loss of self-oscillations close to 1:4 resonance (in Russian). J. Appl. Math. Mech., 42 (1978), 830-840. MR620880 (83e:58064)

14. YU, P.; HAN, M.; YUAN, Y. Analysis on limit cycles of $Z_{q}$-equivariant polynomial vector fields with degree 3 or 4. J. Math. Anal. Appl., 322 (2006), 51-65. MR2238147 (2007f:34060)

15. Zegeling, A. Equivariant unfoldings in the case of symmetry of order 4. Serdica, 19 (1993), 71-79. MR1241150 (94i:58165)

Departament de Matemàtiques i Informàtica, Universitat de les Illes Balears, 07122, PAlma de Mallorca, Spain

E-mail address: chus.alvarez@uib.es

Departament de Matemàtiques, Edifici C, Universitat Autònoma de Barcelona, 08193

Bellaterra, Barcelona, Spain

E-mail address: gasull@mat.uab.cat

Departament de Matemàtiques i Informàtica, Universitat de les Illes Balears, 07122, PAlma de Mallorca, Spain

E-mail address: rafel.prohens@uib.cat 